\title{
The National Cryo-EM Facility
}

Ulrich Baxa, Thomas J. Edwards, Helen Wang, Matt Hutchison, and Sriram Subramaniam

National Cryo-EM Facility, Cancer Research Technology Program, Leidos Biomedical Research, Inc., Frederick National Laboratory for Cancer Research, Frederick, MD, USA

Over the last several years, the field of cryo-EM has undergone a resolution revolution. The data generated by cryo-EM now can be of high enough resolution to directly generate atomic models for proteins and other biological macromolecules. This puts cryo-EM at the same level with other high-resolution structural biology modalities such as X-ray crystallography and nuclear magnetic resonance spectroscopy.

This resolution revolution has been driven by several factors, including advances in microscope and camera technology, as well as improved computational methods for analyzing single particle cryo-EM data. Access to the latest microscope and detector technologies, which can be prohibitively expensive for many institutions is critical for acquiring the best quality data, and generating the highest resolution structures by this method. The NCI has created the National Cryo-EM Facility (NCEF) to meet the needs of cancer researchers in academic labs who do not have adequate access to these instruments.

NCEF currently houses one Titan Krios microscope equipped with a phase plate and a Falcon 3EC direct detector and a K2 Summit direct detector at the end of a Gatan imaging filter. Automated imaging on the $\mathrm{K} 2$ camera is generally performed with Latitude $\mathrm{S}$ (Gatan, Inc.). An upgrade for the K2 summit camera to a $\mathrm{K} 3$ camera is planned and a second similarly equipped Titan Krios microscope will be installed and operational by Fall 2018.

The queue at NCEF is designed to minimize wait time for users, optimize imaging time, and maximize efficiency. To minimize wait time, only one user project from each lab is in the active queue at any one time and data collection is restricted to 48 hours, unless technical problems with the microscope lead to loss of data collection time. Users are encouraged to ship their samples rather than visit in person, so travel coordination can be reduced, and the queue is as flexible as possible in case of instrument issues. Data runs are set up with the users directly engaged via video conferencing. In the future, we plan to have remote access to the imaging computer and on-the-fly feedback on imaging during the run. In the 9 months since opening in May 2017, NCEF has collected data for 27 independent research groups across the US, with $62 \%$ of the time used for user data collection, $15 \%$ for testing purposes and technical development, and $22 \%$ for cryocycles, preventive maintenance, and downtime due to instrument problems. Tracking the time of column valve in the open/closed state allows us to determine that the actual imaging time on days the microscope was being used for user projects was $>85 \%$.

One of the continuing projects at NCEF is to use the general characteristics of the data collected on various projects to better understand trends such as the influence of sample quality on data quality as well as the parameters that affect optimizing collecting the highest quality of images from a given sample. User datasets are not analyzed at NCEF; only for optimizing image quality, and the data is deleted once it has been transferred to the user. A representative example of the type of information we track from individual data collection runs is shown in Figures 1 and 2, with plots reflecting both variations in image quality as well as variations in ice thickness across the data set. As publications that report structures determined at NCEF are published, we will be able to better assess how improvements can be made in procedures for data acquisition [3]. 


\section{References:}

[1] Zhao J et al, J Struct Biol 192 (2015), p. 209.

[2] Feja B, Aebi U, J Microsc 193 (1999), p. 15.

[3] This project has been funded with Federal funds from the Frederick National Laboratory for Cancer Research, National Institutes of Health, under contract HHSN261200800001E. The content of this publication does not necessarily reflect the views or policies of the Department of Health and Human Services, nor does mention of trade names, commercial products or organizations imply endorsement by the US Government.
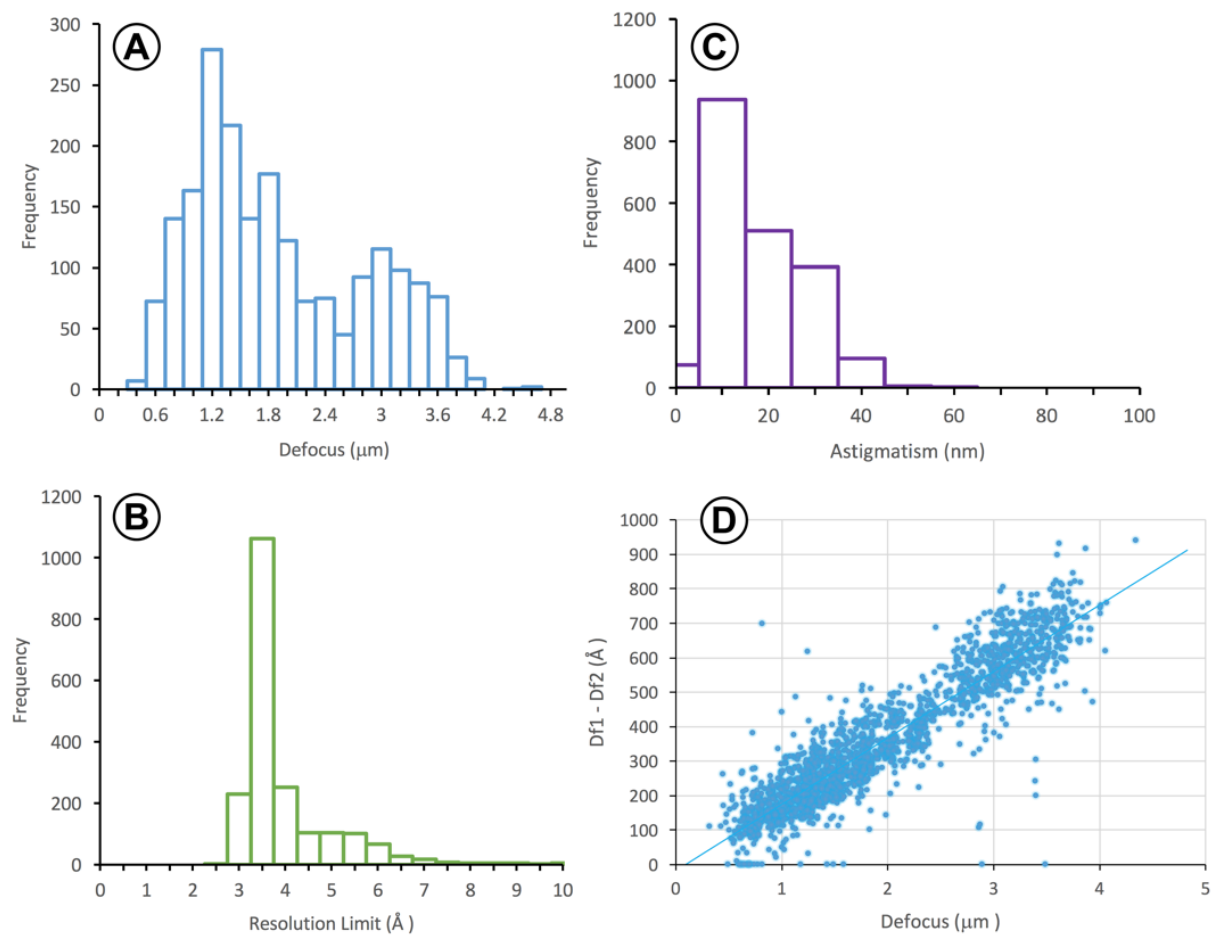

Figure 1. Quality control of a representative data collection run at NCEF. Defocus distribution (A), information limit distribution (B), and astigmatism distribution (C) is shown for all images. (D) shows a plot of the defocus variation (Df1-Df2) over the range of data collected following the approach described in [1]. Automated imaging was performed with Latitude S (Gatan, Inc.).
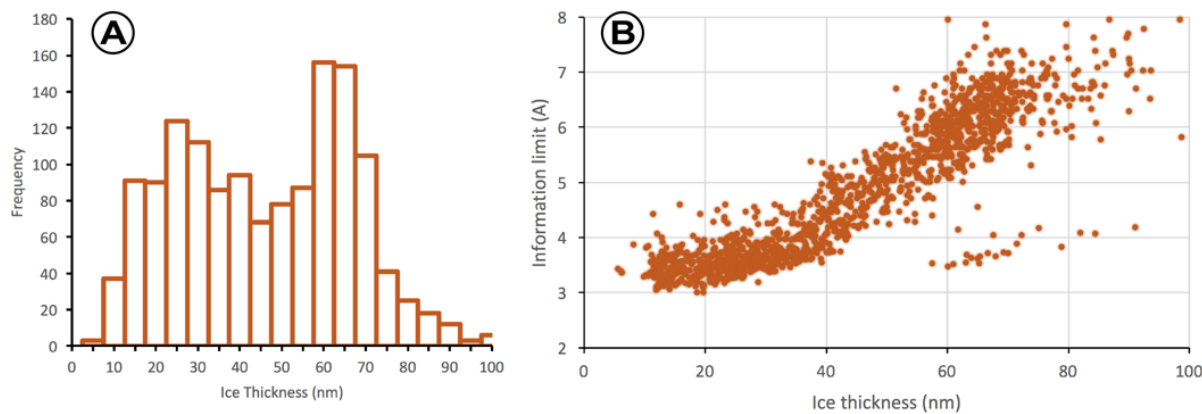

Figure 2. Ice thickness influences the information limit. (A) ice thickness distribution of a specimen imaged in a data collection run at NCEF and (B) plot of the same data showing the variation in the resolution limit at different ice thicknesses for all images. Ice thickness was estimated using the inelastic mean free path measured according to [2]. 